\title{
Unusual Blueshifting of Optical Band Gap of CdS Nanocrystals through a Chemical Bath Deposition Method
}

\author{
Shu Qing Yuan, Peng Fei Ji, Yong Li, Yue Li Song, and Feng Qun Zhou \\ Department of Physics and Solar Energy Research Center, Pingdingshan University, Pingdingshan 467000, China \\ Correspondence should be addressed to Yong Li; liyong@pdsu.edu.cn
}

Received 19 January 2015; Accepted 2 March 2015

Academic Editor: Chang Q. Sun

Copyright (C) 2015 Shu Qing Yuan et al. This is an open access article distributed under the Creative Commons Attribution License, which permits unrestricted use, distribution, and reproduction in any medium, provided the original work is properly cited.

\begin{abstract}
CdS nanocrystals are synthesized through a chemical bath deposition method. After annealing, these nanocrystals are enlarged according to Scherrer's formula. Small nanocrystals display wide band gaps as a result of the quantum effect experienced by nanocrystals of a certain size. However, the absorption edge and green and red emissions of annealed CdS nanocrystals show obvious blueshift compared with the as-grown ones. After annealing, the intensity ratio of these green and red emissions increases, which indicated that the defect states are reduced. Therefore, the improvement in crystalline quality and the reduced strain contribute to the unusual blueshifting of the optical band gap and of the green and red emissions.
\end{abstract}

\section{Introduction}

In electronic and optoelectronic devices, semiconductor nanomaterials have attracted considerable attention because of their unique physical and chemical properties, which are novel and are used more extensively than their bulk counterparts [1]. Among these nanomaterials, CdS is an important II-VI semiconductor that has a direct band gap of $2.42 \mathrm{eV}$ at room temperature. It may be applied to optoelectronics in the visible spectrum range [2]. CdS nanocrystals are grown using various techniques, including thermal evaporation [3], chemical vapor deposition [4], radio frequency sputtering [5], and chemical bath deposition (CBD) [6]. Among these techniques, $\mathrm{CBD}$ is the simplest, coolest, and most economical deposition method for large areas [7]. Small nanocrystals typically display wide band gaps because of the quantum confinement effect experienced by grains of a certain size (less than $50 \mathrm{~nm}$ ) [8]. In this paper, CdS nanocrystals are fabricated through the chemical bath deposition method. In order to obtain the stable hexagonal structure CdS, the annealing treatment is carried out. However, the band gaps of large annealed nanocrystals are wider than those of the asgrown samples. We therefore ascribe the unusual blueshift of the optical band gap to the improvement in crystalline quality and the reduced strains.

\section{Experiments}

CdS nanocrystals were prepared through CBD. The experimental procedure is provided as follows. First, $0.003 \mathrm{~mol}$ cadmium chloride and $15 \mathrm{~mL}$ ammonia ( $25 \%$ to $28 \%)$ were mixed into $60 \mathrm{~mL}$ deionized water. To homogenize the mixture, it was then placed into a bath at a fixed temperature of $80^{\circ} \mathrm{C}$ for $60 \mathrm{~min}$ under magnetic agitation. Furthermore, $5 \mathrm{~mL}$ ammonium chloride $\left(0.1 \mathrm{~mol} \mathrm{~L}^{-1}\right)$ and $5 \mathrm{~mL}$ thiourea $\left(2 \mathrm{~mol} \mathrm{~L}^{-1}\right)$ were incorporated into the mixture. The yellow precipitates reacted for $60 \mathrm{~min}$. They were then filtrated and washed thrice with ethanol. The resultant yellow powders were dried in pure $\mathrm{N}_{2}$ atmosphere. Half of the yellow powder was protected by high-purity argon flow (99.999\%) and annealed for $60 \mathrm{~min}$ at $500^{\circ} \mathrm{C}$. The as-grown CdS nanocrystals have a metastable cubic structure [9].

The morphological and structural properties of the CdS nanocrystals were characterized by high-resolution transmission electron microscopy (HR-TEM, JEM-2100) at an acceleration voltage of $200 \mathrm{kV}$ and X-ray diffraction (XRD, Panalytical X'Pert Pro) with Cuk $\alpha$ as the X-ray source $(\lambda=$ $1.5046 \AA$ ). The absorption spectra of the CdS crystals were obtained using a UV-vis-IR spectrophotometer (Shimadzu, UV-3150) with an integrating sphere detector. Room temperature photoluminescence was measured using a double 


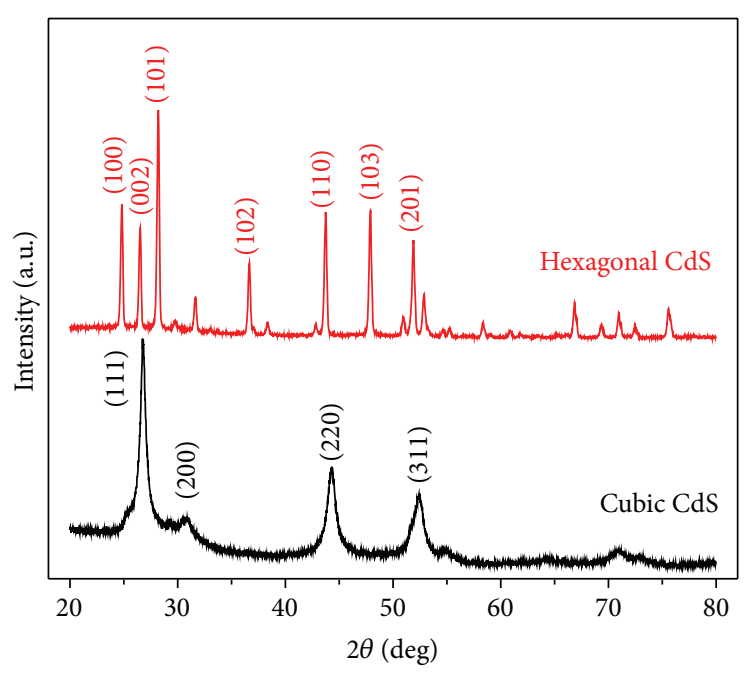

FIGURE 1: XRD spectra of the as-grown (bottom) and (top) CdS nanocrystals annealed.

grating spectrofluorometer (HORIBA, FL3-22), with a Xe lamp as the excitation source.

\section{Results and Discussion}

Figure 1 shows the XRD spectra of the as-grown and annealed $\mathrm{CdS}$ nanocrystals. The as-grown CdS nanocrystals (bottom of Figure 1) predominantly peak once at $26.7^{\circ}$ and correspond to the (111) family planes of the cubic structure (JCPDS: 01-089$0440)$. The three other diffraction peaks at $30.8^{\circ}, 44.3^{\circ}$, and $52.5^{\circ}$ are associated with the (200), (220), and (331) family planes of the cubic CdS structure, respectively. Small cubic $\mathrm{CdS}$ nanocrystals display a relatively wide main peak $\left(26.7^{\circ}\right)$ [10], and the average size of as-grown CdS nanocrystals is $\sim 9.2 \mathrm{~nm}$ according to Scherrer's formula [7]. The cubic structure transforms into a hexagonal one after annealing at $500^{\circ} \mathrm{C}$ in a pure argon atmosphere. Based on the XRD spectrum of the hexagonal CdS nanocrystals (top of Figure 1, JCPDS: 01-070-2553), seven strong diffraction peaks at $24.9^{\circ}$, $26.5^{\circ}, 28.2^{\circ}, 36.7^{\circ}, 43.8^{\circ}, 47.98^{\circ}$, and $51.8^{\circ}$ were indexed to the (101), (002), (101), (102), (110), (103), and (201) family planes of the hexagonal structure, respectively. The diffraction angles for the peaks of the (100), (002), and (101) family planes are used to calculate average grain size according to Scherrer's formula [7]. After annealing, the sample CdS nanocrystals increase to $\sim 17.4 \mathrm{~nm}$ in size.

To investigate the microstructures of as-grown and annealed CdS nanocrystals, the powders were dispersed in alcohol and transferred to the $\mathrm{Cu}$ grid. Figure 2 displays the corresponding HR-TEM images. These images depict numerous zones with distinct lattice fringes. By measuring the distances between fringes, we can confirm the cubic (Figure 2(a)) and hexagonal (Figure 2(b)) structures of the CdS nanocrystals. As stated previously, the cubic structure becomes hexagonal after annealing at $500^{\circ} \mathrm{C}$. This result agrees with the XRD analysis. Moreover, this process also enlarges the CdS nanocrystals. Statistically, the average sizes of the as-grown and annealed CdS nanocrystals are 5.7 and $12.9 \mathrm{~nm}$, respectively (as exhibited in Figure 2(c)). These values are smaller than those obtained through XRD analysis because the HR-TEM images captured a relatively local region, whereas the $\mathrm{XRD}$ analysis gathers information from a much larger area.

To evaluate potential optical properties, the absorption spectra of the as-grown and annealed CdS nanocrystals are obtained and presented in Figure 3(a). In this figure, the absorption edges of the annealed $\mathrm{CdS}$ nanocrystals blueshift more significantly and are steeper than those of the as-grown $\mathrm{CdS}$ nanocrystals, thus indicating that the size distribution of annealed CdS nanocrystals has shrunk. Moreover, absorption in short wavelengths is enhanced after annealing. For direct interband transitions, the following formula calculates the relationship between absorption coefficient $\alpha$ (near the absorption edge) and optical band gap $E_{g}[11,12]$ :

$$
(\alpha h v)^{2}=A\left(h \nu-E_{g}\right)
$$

where $A$ is the parameter related to the effective masses of the valence and conduction bands and $h v$ is the photon energy. Figure 3(b) shows the evolution of $(\alpha h \nu)^{2}$ versus $h v$ according to (1). Optical band gaps also blueshift after annealing, and the calculated optical band gaps of the cubic and hexagonal CdS nanocrystals are 2.398 and $2.461 \mathrm{eV}$, respectively. Under the quantum size effect, the band gaps of small nanocrystals should be less than $\sim 50 \mathrm{~nm}$ wide [8]. However, the calculated band gaps contradict this principle. In as-grown CdS nanocrystals, structural defects and strain should strengthen tailing [13]. Furthermore, annealing can also limit strain. This process can also improve crystalline quality. Thus, the unusual blueshifting of the optical band gap can be ascribed to the improvement in crystalline quality and the reduced strain.

To distinguish this unusual blueshifting, Figure 3(c) depicts the photoluminescence of as-grown and annealed $\mathrm{CdS}$ nanocrystals under an excitation wavelength of $440 \mathrm{~nm}$. In Figure 3(c), the photoluminescence of as-grown CdS nanocrystals displays broad red and weak green emissions 


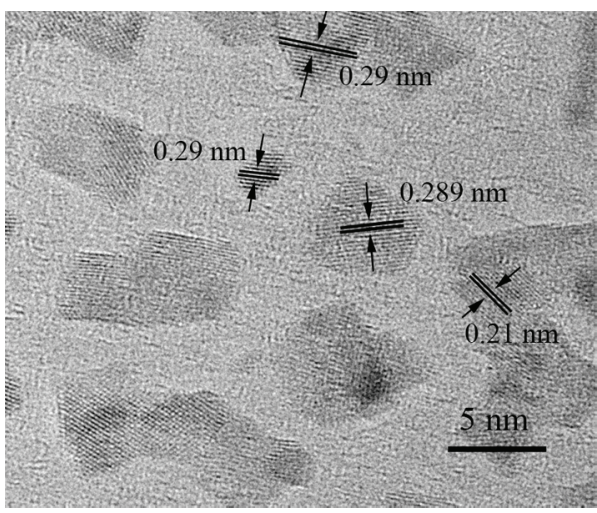

(a)

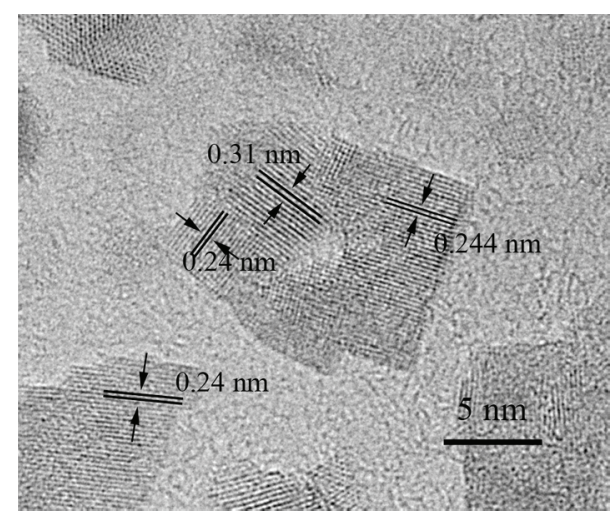

(b)

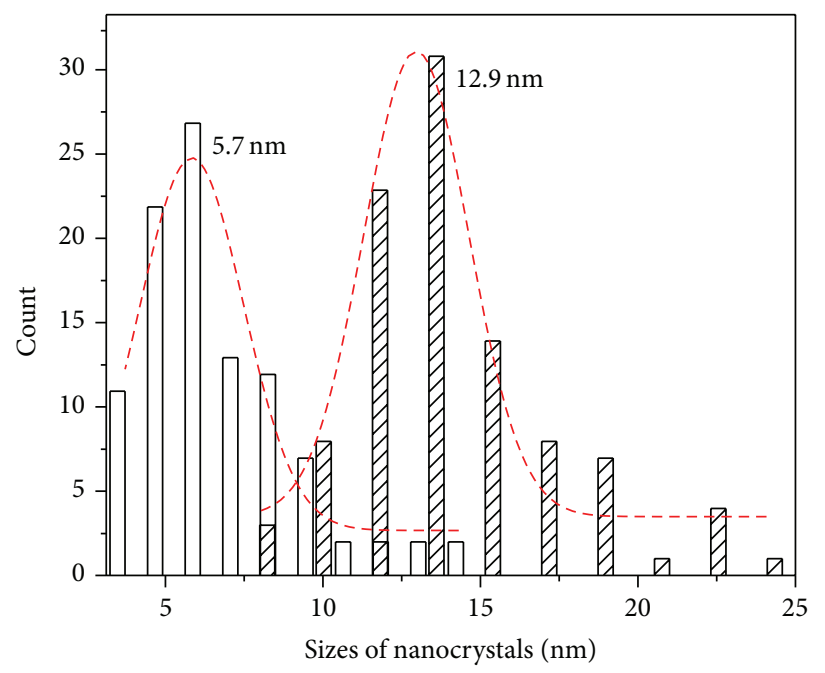

As-grown

WII $500^{\circ} \mathrm{C}$

(c)

Figure 2: HR-TEM images of (a) as-grown and (b) annealed samples. (c) Statistical average sizes.

centered at $\sim 713 \mathrm{~nm}(1.74 \mathrm{eV})$ and $\sim 533 \mathrm{~nm}(2.33 \mathrm{eV})$, respectively. The green emission is ascribed to the emission near the band gap [14]. The absorption peak indicates a large Stokes shift, which suggests that the lattice phonons [15] are strongly related to the size effect [16] of CdS nanocrystals at room temperature. The red emissions of CdS nanocrystals have been studied intensively [14, 17], and these previous studies suggest that red emissions are generally caused by the recombination of surface states to the valence band, impurities and complexes [18-20], and self-activated center [21]. Lee et al. reported that vacancy cadmium $\left(V_{\mathrm{Cd}}\right)$ was located at $\sim 0.7 \mathrm{eV}$ level below the conduction band [22]. In our study, we confirm that the red emission is induced by the transition from $V_{\mathrm{Cd}}$ to the valence band given the calculated optical band gap $(\sim 2.398 \mathrm{eV})$.

After annealing, the normalized photoluminescence spectra show that the green emission blueshifts (the inset of Figure 3(c)), which proves that the optical band gap has been enhanced. The full width at half maximum of the green emissions is lower in the annealed nanocrystals than in the as-grown samples, thus confirming that the size distribution of annealed CdS nanocrystals shrinks and that the surface effect decreases [23]. Furthermore, the intensity ratio of the green and red emissions is much larger in the annealed nanocrystals than in the as-grown samples (Figure 3(c)). This result suggests that defects are limited after annealing. Moreover, the red emission of the annealed nanocrystals blueshifts more significantly than that of the as-grown CdS nanocrystals as a result of the improved band gap. This red emission is caused by the transition from $V_{\mathrm{Cd}}$ to the valence band.

\section{Conclusion}

CdS nanocrystals are synthesized through the CBD method. The size of these nanocrystals increases from $\sim 9.7 \mathrm{~nm}$ to $\sim 17.4 \mathrm{~nm}$ after annealing at $500^{\circ} \mathrm{C}$. Moreover, the absorption edges of the nanocrystals blueshift significantly as a result of reduced structural defects and strain. In addition, the green 


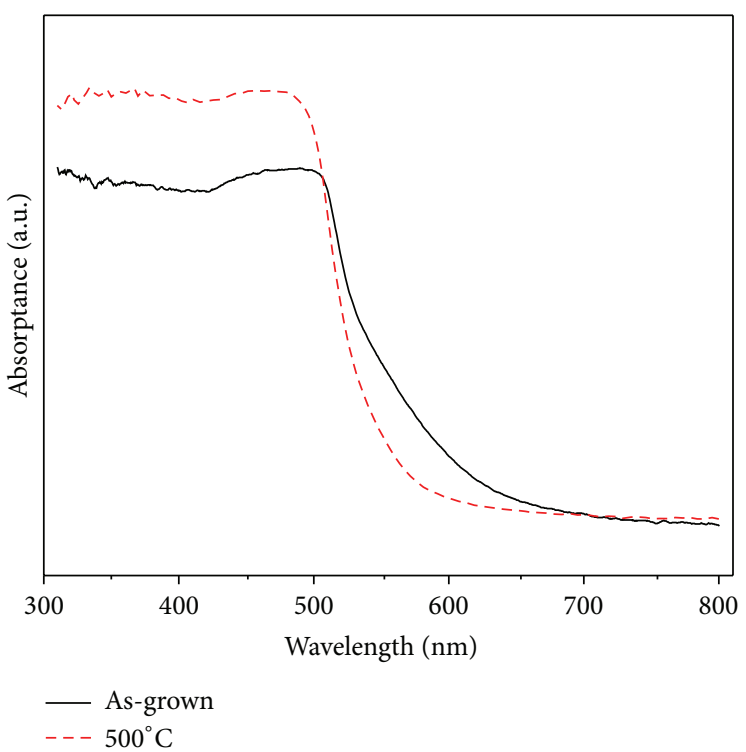

(a)

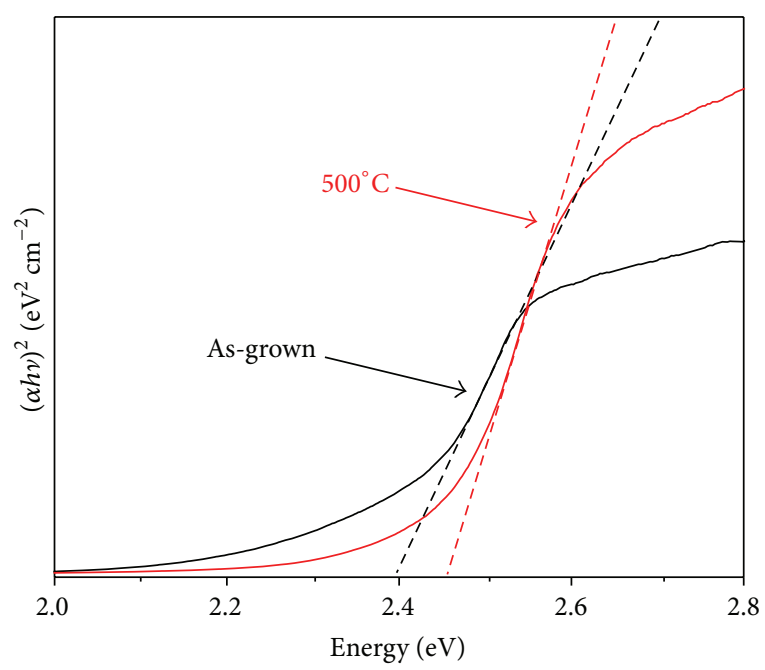

(b)

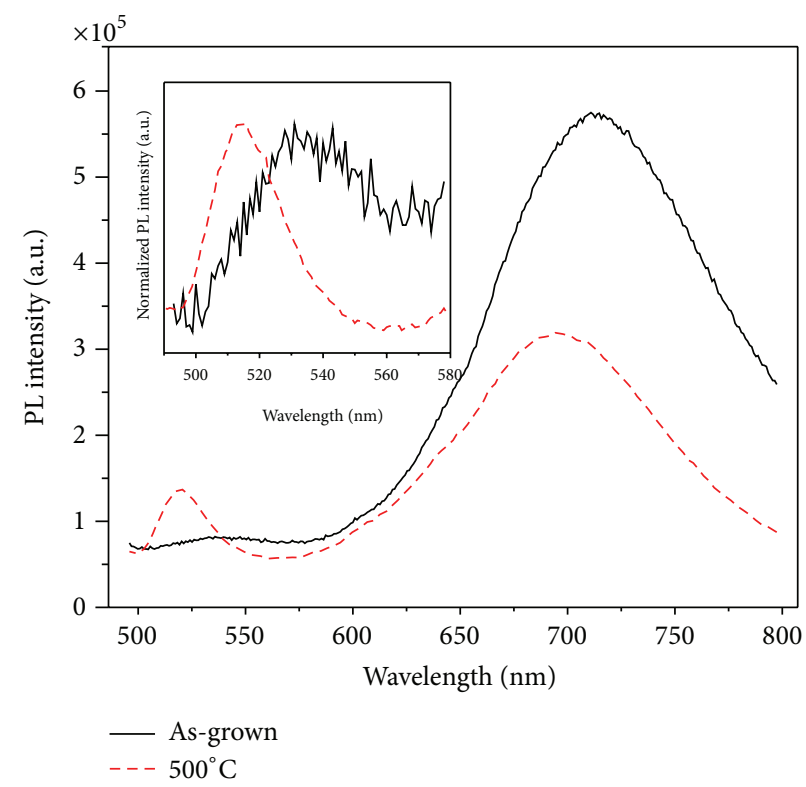

(c)

Figure 3: (a) Absorptance of as-grown and annealed CdS nanocrystals. (b) Calculated optical band gaps. (c) Photoluminescence of as-grown and annealed samples. Inset: normalized PL spectra.

and red emissions of the annealed CdS nanocrystals blueshift more than those of the as-grown samples.

\section{Conflict of Interests}

The authors declare that there is no conflict of interests regarding the publication of this paper.

\section{Acknowledgments}

This work was supported by the National Natural Science Foundation of China (11074224 and 61176044), the Science and Technology Project on Key Problems of Henan Province (082101510007), the Research Project for Basic and Forefront Technology of Henan Province (132300410301), and the Key Research Project for Science and Technology of the Education Department of Henan Province (13B430181).

\section{References}

[1] J. Jie, W. Zhang, I. Bello, C.-S. Lee, and S.-T. Lee, "Onedimensional II-VI nanostructures: synthesis, properties and optoelectronic applications," Nano Today, vol. 5, no. 4, pp. 313336, 2010. 
[2] P. Q. Zhao, L. Z. Liu, H. T. Xue, X. L. Wu, J. C. Shen, and P. K. Chu, "Resonant Raman scattering from CdS nanocrystals enhanced by interstitial Mn," Applied Physics Letters, vol. 102, no. 6, Article ID 061910, 2013.

[3] R. Grover, R. Srivastava, O. Rana et al., "Electroluminescence from hybrid organicinorganic LEDs based on thermally evaporated CdS thin films," Journal of Luminescence, vol. 132, no. 2, pp. 330-336, 2012.

[4] B. Liu, R. Chen, X. L. Xu et al., "Exciton-related photoluminescence and lasing in CdS nanobelts," The Journal of Physical Chemistry C, vol. 115, no. 26, pp. 12826-12830, 2011.

[5] C. T. Tsai, D. S. Chuu, G. L. Chen, and S. L. Yang, "Studies of grain size effects in rf sputtered CdS thin films," Journal of Applied Physics, vol. 79, no. 12, pp. 9105-9109, 1996.

[6] O. Vigil-Galán, A. Morales-Acevedo, F. Cruz-Gandarilla et al., "Characterization of CBD-CdS layers with different S/Cd ratios in the chemical bath and their relation with the efficiency of CdS/CdTe solar cells," Thin Solid Films, vol. 515, no. 15, pp. 60856088, 2007.

[7] S. Kumar, P. Sharma, and V. Sharma, "CdS nanofilms: synthesis and the role of annealing on structural and optical properties," Journal of Applied Physics, vol. 111, no. 4, Article ID 043519, 2012.

[8] Y. Lei, W. K. Chim, H. P. Sun, and G. Wilde, "Highly ordered CdS nanoparticle arrays on silicon substrates and photoluminescence properties," Applied Physics Letters, vol. 86, no. 10, Article ID 103106, 2005.

[9] J. K. Cooper, J. Cao, and J. Z. Zhang, "Exciton dynamics of CdS thin films produced by chemical bath deposition and DC pulse sputtering," ACS Applied Materials and Interfaces, vol. 5, no. 15, pp. 7544-7551, 2013.

[10] L. Kong, J. Li, G. Chen, C. Zhu, and W. Liu, "A comparative study of thermal annealing effects under various atmospheres on nano-structured CdS thin films prepared by CBD," Journal of Alloys and Compounds, vol. 573, pp. 112-117, 2013.

[11] J. H. Warner and R. D. Tilley, "Synthesis and self-assembly of triangular and hexagonal CdS nanocrystals," Advanced Materials, vol. 17, no. 24, pp. 2997-3001, 2005.

[12] R. Mariappan, M. Ragavendar, and V. Ponnuswamy, "Growth and characterization of chemical bath deposited $\mathrm{Cd}_{1-x} \mathrm{Zn}_{x} \mathrm{~S}$ thin films," Journal of Alloys and Compounds, vol. 509, no. 27, pp. 7337-7343, 2011.

[13] E. Rabinovich and G. Hodes, "Effective bandgap lowering of CdS deposited by successive ionic layer adsorption and reaction," Journal of Physical Chemistry C, vol. 117, no. 4, pp. 1611-1620, 2013.

[14] A. E. Abken, D. P. Halliday, and K. Durose, "Photoluminescence study of polycrystalline photovoltaic CdS thin film layers grown by close-spaced sublimation and chemical bath deposition," Journal of Applied Physics, vol. 105, no. 6, Article ID 064515, 2009.

[15] A. Narayanaswamy, L. F. Feiner, and P. J. van der Zaag, “Temperature dependence of the photoluminescence of InP/ZnS quantum dots," Journal of Physical Chemistry C, vol. 112, no. 17, pp. 6775-6780, 2008.

[16] Z. Yu, J. Li, D. B. O'Connor, L.-W. Wang, and P. F. Barbara, "Large resonant stokes shift in CdS nanocrystals," The Journal of Physical Chemistry B, vol. 107, no. 24, pp. 5670-5674, 2003.

[17] Q. Pan, K. Huang, S. Ni, Q. Wang, F. Yang, and D. He, "Fabrication and photoluminescence properties of large-scale hierarchical CdS dendrites," Materials Letters, vol. 61, no. 26, pp. 4773-4776, 2007.
[18] D. K. Dobson, I. Visoly-Fisher, G. Hodes, and D. Cahen, "Stabilizing CdTe/CdS solar cells with $\mathrm{Cu}$-containing contacts to p-CdTe," Advanced Materials, vol. 13, pp. 1495-1499, 2001.

[19] B. Liu, G. Q. Xu, L. M. Gan, C. H. Chew, W. S. Li, and Z. X. Shen, "Photoluminescence and structural characteristics of CdS nanoclusters synthesized by hydrothermal microemulsion," Journal of Applied Physics, vol. 89, no. 2, pp. 1059-1063, 2001.

[20] A. A. Ingale, S. Mishra, U. N. Roy, P. Tiwari, and L. M. Kukreja, "Structural and particulate to bulk phase transformation of CdS film on annealing: a Raman spectroscopy study," Journal of Applied Physics, vol. 106, no. 8, Article ID 084315, 2009.

[21] Y. Shiraki, T. Shimada, and K. F. Komatsubara, "Optical studies of deep-center luminescence in CdS," Journal of Applied Physics, vol. 45, no. 8, pp. 3554-3561, 1974.

[22] H. Lee, H. Yang, and P. H. Holloway, "Functionalized CdS nanospheres and nanorods," Physica B: Condensed Matter, vol. 404, no. 22, pp. 4364-4369, 2009.

[23] X. Xu, Y. Zhao, E. J. Sie et al., "Dynamics of bound exciton complexes in CdS nanobelts," ACS Nano, vol. 5, no. 5, pp. 36603669, 2011. 

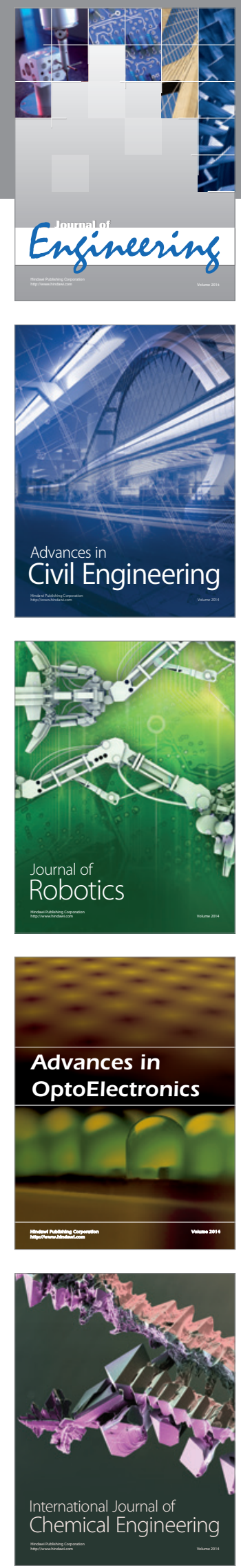

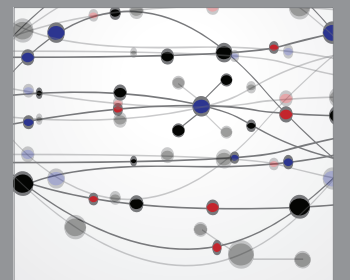

The Scientific World Journal
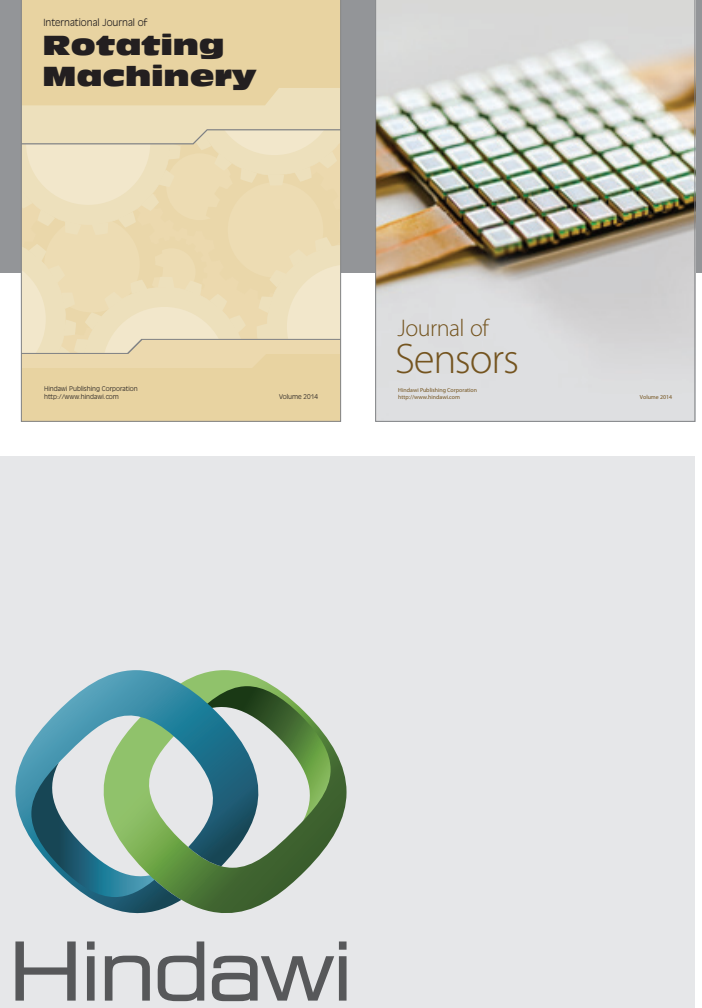

Submit your manuscripts at http://www.hindawi.com
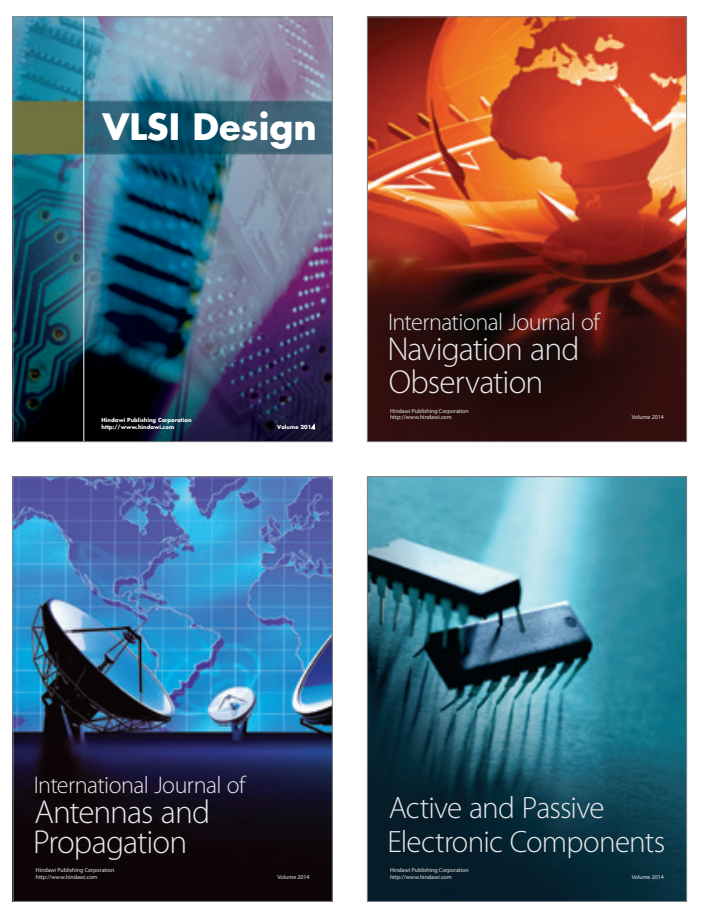
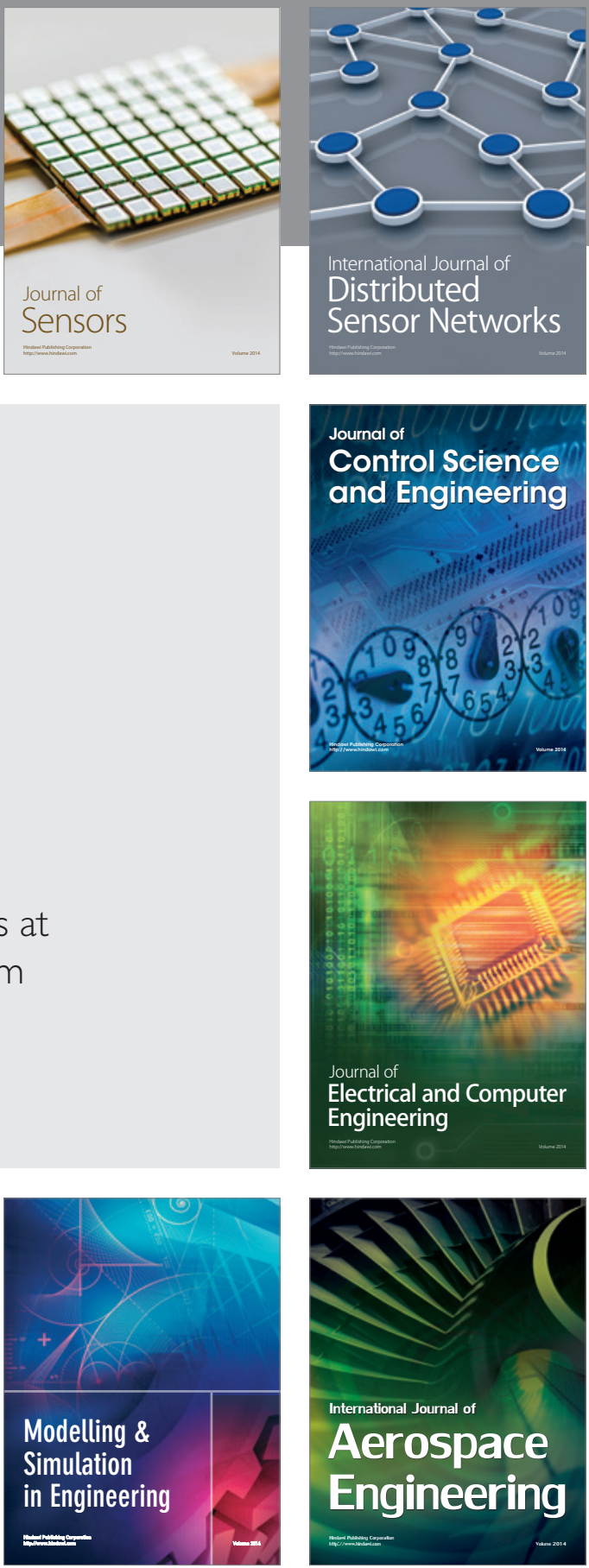

Journal of

Control Science

and Engineering
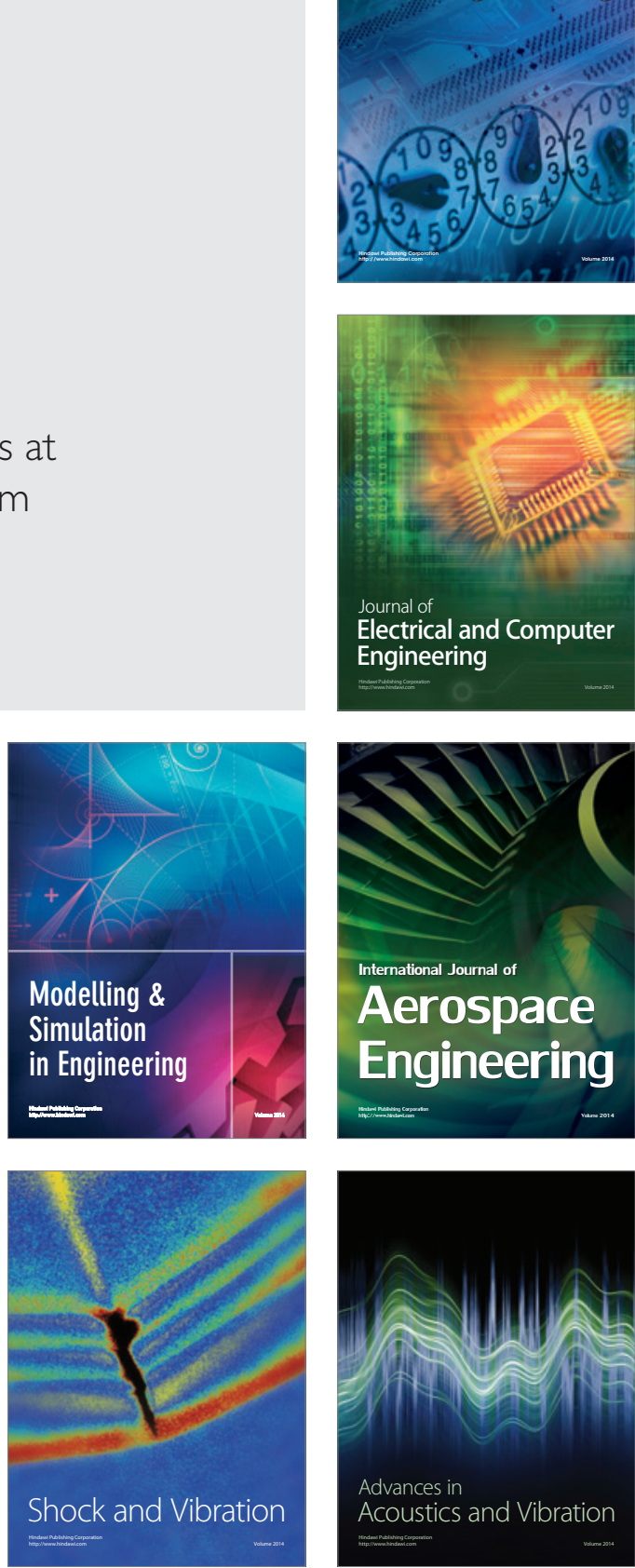